\title{
APROXIMACIÓN AL PAISAJE DESDE UNA NUEVA PROPUESTA: SELF TERRITORY 174, técnica etnográfica aplicada a la interacción paisaje-sujeto y experiencia de salud
}

\author{
Carmen GAONA PISONERO \\ Universidad Rey Juan Carlos (España) \\ carmen.gaona@urjc.es
}

\begin{abstract}
PPROACH TO THE LANDSCAPE FROM A NEW PROPOSAL: SELF TERRITORY 174, ethnographical technique applied to the Landscape-subject interaction and health experience
\end{abstract}

Resumen: Desde nuestro planteamiento reflexivo en el Paisaje se encuentra una proyectabilidad sígnica propiciadora de que nuestras subjetividades puedan seguir cohabitando desde la normalización y la resistencia junto a dialécticas constantes y creativas. Dicha perspectiva será desarrollada en los párrafos siguientes, tras una primera demarcación conceptual del Paisaje desde la que conectaremos dicha proyectabilidad. En una segunda parte del texto, se presenta una nueva herramienta etnográfica para captar y entender la proyectabilidad sígnica del paisaje en comunidades interpretativas locales. En concreto se ha elaborado una nueva técnica etnográfica para entender cómo gestiona, experimenta y reproduce un grupo territorial-cultural su salud desde la interacción Paisaje/subjetividades, mediante la DST: Diagnosis Self Territory.

Abstract: In the way we interpret ourselves in the Landscape, there is a signic projectability that allows our subjectivities to cohabit (in both normalization and resistance) along with constant and creative dialectics.

Such perspective will be developed in the paragraphs below, after an initial conceptual demarcation of the Landscape, from which we will connect the aforementioned projectability. The second part of the text presents a new etnographic tool that lets us grasp and understand the signic projectability of the Landscape of interpretative local communities.

In particular, a new etnographic technique (namely DST: Diagnosis Self Territory) has been developed to understand how a territorial/cultural group manages, experiments and reproduces his own health in the Landscape/subjectivities interaction.

Palabras clave: Paisaje. Cuerpo. Etnografía. Territorio. Imaginario. Salud Landscape. Body. Ethnography. Territory. Imaginary. Health 
"El paisaje es el más rico de los signos"... de toda la vida (Roland Barthes) ${ }^{1}$.

\section{Introducción}

En tiempos no muy lejanos, caminando y aprehendiendo por la ruta GR-92 de Cataluña, fueron frecuentes los letreros informativos que además de señalar datos sobre latitud y kilómetros me narraban cuáles de los caminos eran "senderos saludables". ¿Acaso, los no marcados como saludables, eran insaludables? O dentro de un giro pragmático eran senderos de valores olvidados o secuestrados de nuestro actual momento social, silenciados hasta en su grabado. Sin saberlo había transitado por entre senderos de dignidad, senderos de tolerancia, senderos de creatividad o quizás por ocultos senderos repletos de riesgo para mi salud y subjetividad. Una vez más, constataba cuan ciertas y actuales seguían siendo las palabras de C. Geertz, al advertirnos de que sólo se puede vivir el mundo humanamente desde el sentido que narrativamente el hombre le otorga y el mismo mundo permite. Lo innegable es que es mucho más grato que desde las estructuras de poder nos recuerden la conexión existente entre territorio y salud humana, a que nos medio visibilicen como van mermando los Fondos de Reserva de la Seguridad Social Española, no obstante en ambos casos nos encontramos con dos estrategias biopolíticas diferentes de medicalización. Un proceso de medicalización que también ha adaptado sus sutiles estrategias de control a los nuevos procesos de la cultura y de la subjetividad contemporánea.

Si bien no es objeto de este artículo hacer una exposición teórica sobre dichos procesos de subjetivización, no queremos pasar por alto las reflexiones hechas sobre nuestras subjetividades por parte de André Breton, Lucien Sève, Richard Sennet (2000) y Nikolas Rose (2012), estos dos últimos herederos de una clara influencia foucaultiana. El filósofo francés Lucien Sève, aludía al olvido de nuestra causa antropológica, lo que nos ha encaminado hacia la imposibilidad de vivir en conformidad a lo que constituye nuestra esencia. Frente a la amenaza de Sève, el desespero de Breton ante el hambre de realización espiritual.

Richard Sennett en su obra La corrosión del carácter, desde el supuesto del logro de óptimos niveles de autonomía social de los sujetos, analiza aquellos cambios y transformaciones que tienen una clara influencia con la ansiedad actual provocada por una ausencia de una pauta prefijada para la acción. Los escenarios del mundo laboral, son caracterizados por Sennet como espacios sin rumbo y añade un segundo desconcierto, la falta de anclajes emocionales. Desde estos escenarios caracterizados por la falta de referentes claros, el sujeto no puede detener el ritmo imperante de un continuum de elecciones y riesgos que es la vida. La ansiedad de no saber qué caminos seguir ni las consecuencias que tendrán las decisiones individuales a las que debemos enfrentarnos tanto en el trabajo como en nuestra emocionalidad va erosionando nuestras identidades. Esta aparente mayor autonomía encubre mecanismos complejos, "que tienden a distribuir democráticamente los perjuicios de los caminos erróneos con la consecuente socialización del fracaso, y a concentrar en unos pocos los beneficios del éxito" (Papalini, 2015: 23). Por suerte frente a los caminos erróneos, podemos transitar que no habitar por esos "senderos saludables", y regresar los lunes a nuestro trabajo con una merma de nuestro socializado fracaso, aunque en una aparente alabada libertad corporal. En un escenario sociocultural escrito desde el capitalismo-liberal-libertario

1 La frase "el paisaje es el más rico de los signos" es atribuible a Roland Barthes, la segunda parte es en recuerdo y agradecimiento a Angel Amezcúa Zendejas y Guillermo Hernández García, pues sin duda este artículo no hubiese tomado forma sin su impulso. Mi más sincero agradecimiento a ambos y espero que se reconozcan en este texto, pues parte de nuestras conversaciones y reflexiones están contenidas en él. 
reina en efecto un individualismo narcicista (Lasch, Christopher, 1999) que customiza, que transforma el cuerpo en simple moneda de cambio y lo vuelve apto para seguir sosteniendo y manteniendo el "nuevo espíritu del capitalismo".

En esa misma línea reflexiva sobre las subjetividades actuales pero desmarcándose de la reflexión de R. Sennet, N. Rose, en su obra Políticas de la vida, explora una serie de transformaciones significativas en las contemporáneas "tecnologías de subjetificación" de la vida humana. Para este autor, en el siglo XXI, la política de la vida ya no respondería al principio socializante de la "normalización", sino más bien al modelo individualizante de la customización. Emerge así desde la óptica de Rose una nueva forma de relacionarse con uno mismo, estructurada por el imperativo ético de hacerse cargo activamente de la maximización de la propia vitalidad y calidad de vida. No obstante, no podemos pasar por alto que cualquier iniciativa emprendida desde nuestras subjetividades actuales de customización pese a responder a órdenes de intencionalidad ${ }^{2}$ de diferenciación e inclusive como producto de cierta resistencia, obedece a un primer "acto" de expresión de nuestra identidad corpórea, y que ésta en sus niveles de construcción debe vencer también los niveles de control desde los latentes imaginarios de la sociedad instituida. Además la educación de los cuerpos, que es parte de un proceso de constitución del sujeto, es también una normalización de sus niveles de resistencia y de interiorización de los patrones de exigencia, que deben estar preparados para cohabitar. El cuerpo dice David le Breton (1995) no escapa a la condición que hace de toda cosa propia el hombre el efecto de una construcción social y cultural, dentro de límites infinitamente variables.

Tampoco podemos obviar un elemento imprescindible en la reflexión sobre las subjetividades actuales y los procesos de representación simbólica: el paisaje. Éste tiene un especial protagonismo en la actual construcción de nuestras identidades, tanto desde su presencia e influencia en una primera cognición corpórea, como refugio y destino turístico de las subjetividades fracasadas, y en tercer lugar como escenario principal de ciertas iniciativas de resistencia protagonizadas por algunas comunidades interpretativas ${ }^{3}$. En todos los casos expuestos, desde nuestro planteamiento reflexivo en el Paisaje se encuentra una proyectabilidad sígnica propiciadora de que nuestras subjetividades puedan seguir cohabitando desde la normalización y la resistencia junto a dialécticas constantes y creativas. Dicha perspectiva será desarrollada en los párrafos siguientes, tras una primera demarcación conceptual del Paisaje desde la que conectaremos dicha proyectabilidad; en una segunda parte del texto se presenta una nueva herramienta etnográfica para captar y entender la proyectabilidad sígnica del paisaje en comunidades interpretativas locales. En concreto se ha elaborado una nueva técnica etnográfica para entender cómo gestiona, experimenta y reproduce un grupo territorial-cultural concreto su salud desde el paisaje, mediante la DST: Diagnosis Self Territory. Dicha técnica presentada en este artículo desde una aproximación teórica-empírica se basa en una animalidad de nuestra subjetividad, ambas construidas desde el valor del lenguaje simbólico, en la línea de autores como Paolo Virno (2013). Desde el Self Territory 174 se suma la comprensión de nuestra animalidad, de nuestra salud, de nuestra realidad actual integrando la constitución de significado desde la interacción Paisaje-subjetividades. En definitiva se intentaría añadir a la fusión entre naturalismo e historia, el lenguaje simbólico, recuperando el trabajo etnográfico.

\footnotetext{
2 Desde la expresión de Daniel Dennett

3 En un listado interminable a modo de ejemplo se menciona la Red Natura 2000 en Europa compuesta por más de trescientas asociaciones en defensa del paisaje e implementando acciones concretas de resistencia: AEMSRíos con Vida; Amigos de los Humedales del Sur de Alicante, Plataforma contra el Francking en Guadalajara, Plataforma contra la Especulación Urbanística y Ambiental de Candeleda, Plataforma de Toledo en Defensa del Tajo, Plataforma en Defensa de l'Ebre, etc.
} 


\section{El paisaje en relación con la cultura y las corporeidades: ¿dónde está el paisaje?}

Antes de presentar las relaciones que anuncian el título de este epígrafe, cabe precisar cuándo dejamos de hablar de territorio y descubrimos el paisaje desde nuestra culturalidad.

El paisaje es conceptualizado por algunos como escenarios naturales percibidos en una relación estética; otros hacen notar la presencia del hombre en el cambio de estos escenarios naturales convirtiéndolos en un producto cultural u objeto de humanización (Covarrubias, Cruz y otros, 2011. Daniels y Cosgrove, 1988. Kahn, 1996. Knapp y Ashmore, 1999), autores todos ellos que plasman y reflexionan el paisaje desde diferentes ángulos pero como un producto cultural. Destacamos la conjunción entre paisaje y sujeto hecha por el profesor Covarrubias, quien nos recuerda como la dialéctica marxista piensa al hombre como parte de la naturaleza y a la naturaleza como objeto de humanización. Otra delimitación conceptual del paisaje incide en la relación de éste con la memoria (Criado y Díaz-Fierros, 1986), (Gosden y Lock, 1998. Parcero, Criado y Santos, 1998a , 1998b. Holtorf, 2000-2007), autores todos ellos que nos presentan una significación del paisaje en tanto que construcciones simbólicas y significativas, que han sido depositarias de diferentes memorias culturales. Este planteamiento conectaría con el concepto de Pierre Nora (1984) "lugares de la memoria", si bien para Nora el paisaje compartiría esta función con otros lugares como un archivo, un museo o el festejo de una fiesta tradicional entre otros. En esta línea estaría también el término "memory landscape", significado este último que acentúa "las cualidades mnemotécnicas tanto de los elementos monumentales o señales físicas permanentes del entorno, como de manifestaciones menos tangiles o perdurable (nombre, sitios históricos, paisajes naturales...), que permiten que un grupo reconozca el sentido colectivo de determinados espacios o lugares" (Álvarez, 2010: 179).

En líneas generales, la aproximación teórica al paisaje, pese a incluir su valor sígnico, no presenta al paisaje en toda su globalidad pues lo reduce a un mero repositorio, un refugio de nuestra memoria colectiva o en su relación identitaria. Recogiendo todo el sustrato reflexivo existente, queremos dar un paso más conectando el paisaje no sólo con nuestra memoria colectiva, sino con todo nuestro imaginario social. En un segundo orden de intencionalidad queremos visibilizar (tanto a nivel espistemológico como empírico) la proyectabilidad inherente del paisaje, no en tanto su contención sígnica sino en su forzosa intercomplementariedad con las subjetividades, centrándonos en la experiencia de salud de estas subjetividades junto con sus tácticas de resistencia. La proyectabilidad del paisaje reactiva nuestra entropía social mediante la que se rompe la obediencia, afectando por tanto a cuestionar las facticidades de la medicalización, y también nos conecta con nuestra propia creatividad. En tercer lugar, más allá de lo que propicie a las subjetividades, queremos acercarnos al paisaje desde su propia acción. Nuestras acciones sociales son performativas y en la más absoluta dialéctica en tanto que danzan al mismo son, lenguaje, sujeto espacio, tiempo y poder.

De todo lo expuesto se deduce que en nuestra reflexión el paisaje vaya más allá de su materialidad o a su delimitación geográfica administrativa, dimensión esta última innegable pues la idea de paisaje conlleva también la exigencia de cualificación final de toda intervención sobre cualquier territorio ${ }^{4}$. El paisaje como construcción cultural no se limita a "una entidad tangible formada por elementos biogeográficos (tierra, vegetación, agua, luz, parcelación, explotación económica, urbanismo...), sino que estaría sujeta a un proceso de percepción y valoración personal y social que lo configuran como construcción colectiva" (Watsuji, 206: 24). Por consiguiente a lo material le debemos de sumar lo cultural para empezar a hablar de Paisaje, una unión no gratuita sino resultado de la práctica comunicativa humana, que posibilita que dicha extensión, como conjunto, más que una cosa en sí o una construcción visual y mental, propicie y proyecte nuevas formas de habitar. La acción de

4 Ver Zoido Naranjo, 2006, 2010 a), donde se describen los planteamientos que vinculan paisaje y ordenación del territorio. 
habitar nos remite a su vez a un conjunto de acciones como convivir, comunicar, crear, consensuar, resistir y proponer entre otras acciones más que dan sentido al verbo habitar integrando a su vez las tres dimensiones de lo urbano señaladas por Henri Lefebvre:

"Lefebvre diferencia tres funciones en interrelación constante: la transfuncional, la multifuncional y la lúdica. La dimensión transfuncional se sitúa en el campo del valor simbólico, semiológico y semántico de la monumentalidad o expresión de la creatividad colectiva y de la tensión utópica de la ciudad. La segunda o multifuncional se expresa en la "calle", entendida como lugar de encuentro e intercambio y teatro espontáneo de sociabilidad. Por último, la función lúdica es definida como el momento omnipresente difundido en el espacio de la ciudad, más allá del tiempo y del comportamiento recreativo allende del trabajo" (Gaona, 2009: 98).

Llegados a este punto, ya podemos dar una primera respuesta a nuestro interrogante, ¿dónde está el paisaje? Al igual que montes, llanuras y demás depresiones anteceden a la Cartografía y la Geografía, luego después antes del paisaje existe el territorio, pero exento éste último por completo del más mínimo valor sígnico. Construimos simbólicamente el Paisaje en un primer acto de habla, para pasar luego después en ese diálogo paisaje-sujeto a tener consecuencias directas con distintos ámbitos de nuestra vida. No obstante, nos centraremos en el plano concreto de nuestros procesos de salud, en una interrelación (paisaje y salud) cuya aplicación y reflexión es una constante multidisciplinar. En planteamientos actuales autores como Melanie Rudd, Colin Ellard, recuperan la concepción (derivada de dicha interrelación) "que la exposición a escenas de grandeza, ya se trate de fenómenos naturales asombrosos como un cielo oscuro estrellado o los desfiladeros del Gran Cañón del Colorado o de un artefacto de construcción humana, como el techo de una catedral, pueden ejercer una influencia cuantificable en la concepción que tenemos de nosotros mismos, en cómo tratamos al prójimo e incluso en cómo percibimos el paso del tiempo" (Colin Ellard, 2016: 15). Estas propuestas suponen una vuelta a planteamientos visibilizadores del papel del paisaje en la construcción del individuo, y en los que los individuos no son vistos como seres portadores de unas historias y narraciones pasadas, sino que ese pasado también ha estado conformado (y para algunos autores determinado) por el clima y el paisaje (Watsuji, 2006). En lindes concretos disciplinarios como la Geografía Humana, destacamos autores como Alexander von Humboldt, Paul Vidal de la Blache y Manuel Terán entre otros más. Cabe mencionar también la llamada "Arqueología del Paisaje" 5 con figuras representativas como la obra de Criado Boado (1999), Barret (1999ª 199b), García Sanjúan (2000).

Aunque ya hemos encontrado al paisaje, no por eso podemos dejar de nombrar uno de los principales aportes al estudio del paisaje y por el que se ha definido en ciertas épocas: desde la trayectoria artística del paisaje. Para algunos teóricos (Claudel, 1934. Clark, 1971) el paisaje es materializado desde la mirada pictórica. Es innegable, que si detenemos nuestra mirada en las obras de arte y el ojo llega al alma (como nos propone Paul Claudel) estas obras nos conducen al interior de la naturaleza, pero como veremos más adelante, en realidad nos retorna a un especial refugio de las subjetividades, y digo especial pues tanto sobrevienen en él anomia y destierro como resistencia y salud.

\section{Paisaje y subjetividades: miradas en verbo}

En líneas sucesivas desarrollaré la proyectabilidad sígnica del Paisaje, partiendo de esa

5 Consultar el artículo de Almudena Orejas (1998): "El estudio del paisaje: visiones desde la Arqueología”, en Rev. Arqueología Espacial 19-20, 9-19, Teruel, para una exposición de las principales líneas temáticas de análisis desde una Arqueología del Paisaje. 
unión indisoluble entre Paisaje y subjetividades nombrada en párrafos anteriores. Más allá del plano reflexivo, para percibir y sentir dicha unión se invita al lector a un sencillo ejercicio visual, visitando la propuesta visual del artista José Manuel Ballester en Espacios ocultos $^{6}$, proyecto en el que despoja a obras clásicas -en especial del Renacimiento italiano (selladas con un gran homocentrismo)- de cualquier presencia humana, hasta el punto de desaparecer ésta de la composición pictórica, quedando únicamente el espacio arquitectónico o paisajístico en el que se enmarcaba. Tras visionar estas propuestas de Ballester, ¿ha desaparecido realmente de la mente del lector la proyección inferencial de la figura humana? Es imposible hacer desaparecer al ser humano del paisaje, del espacio. Al igual que no podemos hablar sólo de comunicación, sino de proceso comunicativo, ó de salud sin la experiencia de salud. Del mismo modo, no podemos hablar sólo de Paisaje, de lugar, de espacio, sino de la percepción de la realidad que los provoca, y en la percepción está presente el ojo humano (nuestras subjetividades). Además, el paisaje en tanto que acción, es acción cohabitada con unos de sus principales ocupantes: nosotros. En esta línea sirvan las palabras -claras y rotundas- de Carmen Laffón en su Discurso Visión del Paisaje, con motivo de su entrada a la Real Academia de Arte de San Fernando.

"Desde hace varios años trabajo en una serie de cuadros, que titulo Vistas del Coto. La decisión de que mi discurso trate sobre el tema de éstos ha estado motivada por lo mucho que significa para mí la realidad que los provoca: la desembocadura del Guadalquivir" (Laffón, 2000).

Las palabras de la pintora Laffón, en este juego de doble mirada se asemeja al planteamiento de Javier Maderuelo (1997) cuando señala que "la idea de paisaje se encuentra tanto en el objeto como en la mirada. No es lo que está delante sino lo que se ve" (Maderuelo, 1997: 10). Sirva también la reflexión del profesor Juan Bosco Díaz-Urmeneta, en esa línea reflexiva sobre la indisolubilidad Paisaje y subjetividad: "El lugar es aquello que crece con nosotros, son sitios que crecen con nosotros, van creciendo a la vez que crece quien vive en ellos [...] El sentimiento del paisaje, la paradoja del paisaje que siendo un trozo de la naturaleza nos da la naturaleza entera .. Por eso, los grandes paisajes tienen un poder simbólico"7, a lo que añadimos que en ese poder simbólico también está el reconocernos en él aún sin estar nuestra imagen, pero si está proyectada en el sentir de la mirada.

Pero esa mirada, es una mirada en verbo. En el proceso de socialización el individuo experimenta una identificación progresiva con el Paisaje. Se establece entre ambos una comunicación vis a vis; en tanto el Paisaje es una totalidad autopoiética, , un organismo vivo e integrado en el cosmos, en la realidad social y en los imaginarios colectivos. Una totalidad que nos configura y con el que estamos en diálogo constante, pues la historia nos constata "la presencia de un proceso de retroalimentación en el que los hombres han conformado el medio natural pero al mismo tiempo éste los ha conformado a ellos" (Alvarez Munárriz, 2015: 421).

\section{Paisaje y proyectabilidad sígnica}

El Paisaje es entendido como producto y potenciador de la relación mente/cuerpo, desde la estructuración inferencial de la experiencia del sujeto y de la colectividad. En efecto, el sujeto en su primera identidad corpórea, en tanto que totalidad orgánica y reflexiva, , es un organismo vivo e integrado en el cosmos. En su andadura sociocultural, dicha identidad

6 En: http: //www.josemanuelballester.com/espanol/exposicionEspaciosOcultos/exposicionEspaciosOcultos1. htm

7 Entrevista en el Programa La mitad invisible: Los paisajes en Carmen Laffón, en http: //www.rtve.es/alacarta/ videos/la-mitad-invisible/lmi-sanlucar-avance/3308529/ 
corpórea habita, moldea, lucha, devasta y resignifica el Paisaje, sin poder borrarse de su digamos ADN cultural que las "las civilizaciones son espacios" (Braudel, 1968: 180). Tal y como señala el antropólogo Luis Álvarez Munárriz y respaldando la propuesta de Merleau Ponty sobre el protagonismo de nuestra corporeidad, todo sujeto es un "sí-mismo corporeizado que se relaciona de manera dinámica y creativa con un entorno específico", a lo que debemos añadir que todo entorno es culturalmente construido e histórico. Pero nuestra reflexión, tiene como punto de partida, no una historia de las variaciones categoriales de las producciones culturales de dicha relación de cuerpo y entorno, sino un intento de alcanzar el punto de partida en el que esa totalidad orgánica empieza a ser reflexiva como resultado de la absorción de la información que proporciona el Paisaje, en definitiva exponer teóricamente la proyectabilidad sígnica del paisaje. En un segundo orden nos interesa localizar e interpretar los sentidos presentes en ese dialéctico diálogo Paisaje-subjetividades, que repercuten en sus construcciones identitarias, así como en sus procesos de salud. No sólo el sujeto absorbe del medio nutrientes, aire, sol, agua entre otros elementos más, sino que en primer lugar absorbe también "información" del medio, y en segundo lugar existe un diálogo Paisaje-sujetos. De este modo hablamos del conocimiento corpóreo desde la óptica de Wilson (2002) para quien el entorno es parte del conocimiento cognitivo. El flujo de información entre la nuestra totalidad orgánica y reflexiva y el mundo es tan denso y continuo que, para los científicos que estudian la naturaleza de la actividad cognitiva, la mente por sí sola no es una unidad significativa de análisis.

Por ese motivo, entendemos el inicio de nuestras representaciones y de nuestras narraciones desde la cognición corpórea ${ }^{8}$. Al adoptar la actitud más abstracta de la cognición corpórea, las preguntas sobre la relación mente-cuerpo, sólo se pueden responder desde la concurrencia plena de la mente y el cuerpo (Jonson, 1991. Gaona, 2009). Dicha concurrencia impide que la pregunta qué es la mente se descorporice. Pero desde un planteamiento comunicativo de la cultura, nuestro sí-mismo corporeizado necesita ser accionado por los esquemas de imagen existentes en el Paisaje/medio, y desde éstos empezar su producción de la metáfora conceptual: "La cognición corpórea es sensible al contexto comunicativo en que se produce, una frase u oración, tanto como a los contextos experienciales en que uno interactúa con esa clase de entorno" (Glenbert y otros, 2008). Sin perder de vista que las metáforas se producen y retroalimentan en nuestros imaginarios sociales.

¿Qué es habitar el paisaje? No sólo trabajarlo, cultivarlo, manipularlo sino desde él dar sentidos. ¿Qué es dar sentido? Reproducir las representaciones del mundo desde el imaginario colectivo y desde nuestro universo simbólico. EL Paisaje, POSEE el OBJETO, sin conocerlo, por consiguiente en el paisaje no existe el signo, meramente su significante. El sujeto que HABITA el paisaje mediante la dominación/observación/control/producción sígnica del objeto y aprehensión reflexiva del objeto LO CONOCE, y al conocerlo y habitarlo LO POSEE. Lo posee dentro de la intersubjetividad y consenso colectivo y, en la más absoluta dialéctica creativa al producirse a su vez este proceso desde la conexión inconsciente a nuestros imaginarios sociales, desde los cuales la elaboración y distribución generalizada de instrumentos de percepción de la realidad social se construyen como realmente existentes.

El mundo no se distingue de mí como una suma de cosas o procesos vinculados por unas relaciones de causalidad, o me conecto al mundo desde unas relaciones de producción. Éstas existen, pero coexistiendo con ambas hay un giro pragmático, por el que en un primer orden el mundo lo capto en mí desde mis sentidos, el paisaje lo fagocito en mí desde mis sentidos (lo produzco artísticamente en mí), en un segundo orden, lo resignifico en mi

8 Tradicionalmente una perspectiva objetivista ignoraría el cuerpo al considerar que éste introduce elementos subjetivos que presuntamente no tienen que ver con la naturaleza objetiva del significado. Además, se ha ignorado al cuerpo, porque se pensaba que la razón era abstracta y trascendente, es decir, que no estaba vinculada a ninguno de los aspectos corporales de la comprensión humana 
(desde mi animalidad y desde mi imaginario colectivo) y en un tercer orden lo legitimo para que quede sellado y registrado en mi imaginario de referencia. No hablamos de lugares subjetivos de registro, no hablamos de niveles subyacentes de mi universo simbólico, sino que hablamos del imaginario social, que convive tal y como nos alertó Castoriadis en dialéctica constante con el imaginario hegemónico de lo instituido.

Qué es lo que nos vuelve a conectar con la fuente primigenia de nuestra culturalidad, con la Ausencia (el arte, el verso, Dios): el mundo de la naturaleza que desde el preciso instante en que es mirado en verbo y es habitado, deja de ser territorio y pasa a ser paisaje. El paisaje, que nos devuelve y recuerda constantemente (junto a otros pocos catalizadores ó regeneradores más) la necesidad de volver a hablar en verso, igual que en el Paraíso, representa la capacidad de recordar en forma activa la Ausencia: no buscarla en el pasado ni esperarla en el futuro, sino hacer vivir el recuerdo en nuestro instante presente. De este modo, podremos crear de forma colectiva el momento mágico. La magia reside en hacer desaparecer nuestros límites sociales o distrofias de la percepción, entre ellos el logocentrismo.

En realidad ese volver a hablar en verso, al que nos proyecta el Paisaje, es desprendernos del logocentrismo que a su vez sigue una tendencia centralizadora. Esta tesis está contenida en la teoría de la deconstrucción de Derrida, quien afirma como la humanidad ha creado conceptos que muestran una tendencia centralizadora, dinámica que se constata en lexemas como "esencia", "verdad", "presencia", "dios", "palabra", etc. Dicha tendencia centralizadora no debe confundirnos, pues en realidad tal y como nos dice Derrida es el logocentrismo, ese deseo "no consumado" por conquistar el centro, es el que nos ha llevado a una lógica binaria como presencia/ausencia, palabra/escritura, consciencia/inconsciencia, hombre/ mujer. También en el logocentrismo está esa construcción binaria respecto al movimiento del cuerpo en el espacio, dentro/fuera, arriba/abajo, derecha/izquierda. Pero es justo en la aproximación y construcción cultural del Paisaje, que se destruye dicho logocentrismo, mediante dos dinámicas o dos tácticas. En primer lugar por el hecho de que no estamos ni dentro ni fuera del paisaje, pues como la propia expresión "pisar firme" enuncia: queremos estar dentro del paisaje, formamos parte de él pues ante nuestra ausencia éste desaparece y pasa a ser mero territorio, y además estamos fuera de él puesto que no fusionamos nuestra materialidad ni con sus rocas ni por entre sus manantiales; no se disuelve nuestra corporeidad en y con el territorio. Mucho más explicativas son las palabras del profesor Covarrubias, sobre esa no fusión entre las esencias del paisaje y el sujeto:

"La dialéctica marxista es una postura ambientalista que considera al hombre parte de la naturaleza y transformador de ella por medio del trabajo social. Para satisfacer sus necesidades, el hombre transforma la naturaleza transformándose a sí mismo. El trabajo, en cuanto proceso de apropiación de la naturaleza por el hombre, es materialización del sentido con el que la naturaleza aparece en la conciencia humana, pero que nunca se integra existencialmente a ella sino que permanece en la conciencia confundida con lo real. La naturaleza no tiende direccionalidad en sí, sin embargo el hombre ve en ella un sentido evidente que es producto de la sobreposición a lo real del contenido de su conciencia, como sucede con las supuestas leyes a cuyo dominio se encuentra sometida la naturaleza" (Covarrubias, 2011: 35).

Como segunda "táctica", la localización geográfica del sujeto en el Renacimiento se inestabiliza y le aboca a replantear su ubicación geográfica no dentro del logocentrismo, sino lejos de una lógica binaria y entrar en una tridimensionalidad en la que danzan el cosmos, la divinidad y el sujeto bajo la atenta observación de la ciencia: 
"Las teorías de Copérnico (1473-1543) invalidaron la visión de la Tierra como el centro del universo. A pesar de que éstas fueron prohibidas con el Índice de 1616, el sujeto renacentista ya había captado la inestabilidad de su localización geográfica en el cosmos. Más aún, ubicar a Dios y que éste los ubicara a ellos era su preocupación primordial. Gracias a los descubrimientos científicos del telescopio en 1609, se pudo comprobar que la Tierra giraba en torno al Sol. Estos cambios epistemológicos trajeron como consecuencia un énfasis en la localización espacial del sujeto con respecto al cosmos y en su relación con la divinidad" (Saldarriaga, 2006: 11-12).

Es justo a través de estas dos "tácticas" que el paisaje sin contener el signo si consigue su deconstrucción, y es en dicha posibilidad de salir del logocentrismo que radica la proyectabilidad sígnica del paisaje que no es accionada por un "ego trascendental" (conciencia individual solitaria) de Husserl (1964: 50), o de un nosotros trascendental. Ambos conceptos son estructuras puramente mentales y accesibles a la conciencia, "nosotros o ego trascendental", en un acto de "introspección filosófica abstracta". Me estoy refiriendo a una proyectabilidad sígnica del paisaje activada por subjetividades, por ciudadanos reales sin ocultar esa corporeidad o identidad corpórea presente en todo proceso sociocultural. El propio Henri Lefebvre, en su obra La Production de l'espace, dedica varios momentos a la reflexión del cuerpo y tras exponer la negación del cuerpo en la tradición judeocristiana, traslada la cuestión de la negación e inclusive mutilación simbólica del cuerpo en su auténtica problemática actual, puesto que "ha llevado la división del trabajo hasta el interior mismo del cuerpo de los trabajadores y aun de los no trabajadores" (Lefebvre, 1974: 235236). A lo largo de este texto este mismo autor defiende la tesis de la inventiva del cuerpo, pero siempre en unión con el espacio: "La energía del cuerpo participa así de la creación de un espacio "diferencial", que se opone al espacio abstracto que sirve de instrumento de dominación" (Lefebvre, 1974: 427).

Haciendo un alto en nuestro recorrido reflexivo, quisiera hacer una puntualización, pues el lector puede pensar que pese a querer delimitar conceptualmente la proyectabilidad sígnica del paisaje, estamos dando vueltas y vueltas a nuestra fantasía de investigador. En este sentido sería la misma primera reacción que tenemos cuando nos empiezan a explicar las "Máximas de Grice" y queremos se nos precise el espacio material dónde verlas, para llegar a dislumbrar que pese en un nivel subyacente llevamos miles de años utilizándolas. Por otro lado, al hablar de proyectabilidad sígnica estamos trasladándonos a planteamientos ya existentes y elaborados desde diversos y dispares enfoques, tales como el de Sor Juana Inés de la Cruz, Frederic Jameson o Michel Certeau, que ya abordaron en qué medida la discursividad se ve afectada por la forma de actuar en un espacio y el movimiento del cuerpo dentro de una localización determinada, dentro de un paisaje concreto.

Las fichas que he elaborado y que se presentan en el epígrafe siguiente, están hechas con el objetivo de servir de herramientas detectoras, descriptivas e interpretativas de las distancias que la comunidades locales analizadas marcan respecto a la discursividad normativa de los espacios, alejándose, reproduciendo o denunciando dicha discursividad. El propio Certeau habla de "tácticas" de los cuerpos en su retórica con el espacio, cuando éstos buscan y expresan un deseo de subversión.

\section{Primera aproximación al Self Territory 174}

En esta nueva técnica de análisis social, se suman elementos de la epidemiología, la etnografía y la pragmática. En la intersección de estas tres áreas, se encuentra el potencial del Self Territory 174, para alcanzar una mayor comprensión de la compleja gestión biopolítica y estética de la salud, que realizan grupos culturales con una marcada identificación identi- 
taria, y en comunidades en los que confluye además una fuerte interacción con el patrimonio cultural histórico-medioambiental. A continuación se presentan las cuatro fichas que configuran la técnica de análisis etnográfico Self Territory 174, que surge desde una conceptualización del paisaje definida por la interacción creativa y dialéctica de sus subjetividades en conexión constante con el imaginario social y bajo la lógica de la proyectabilidad sígnica.

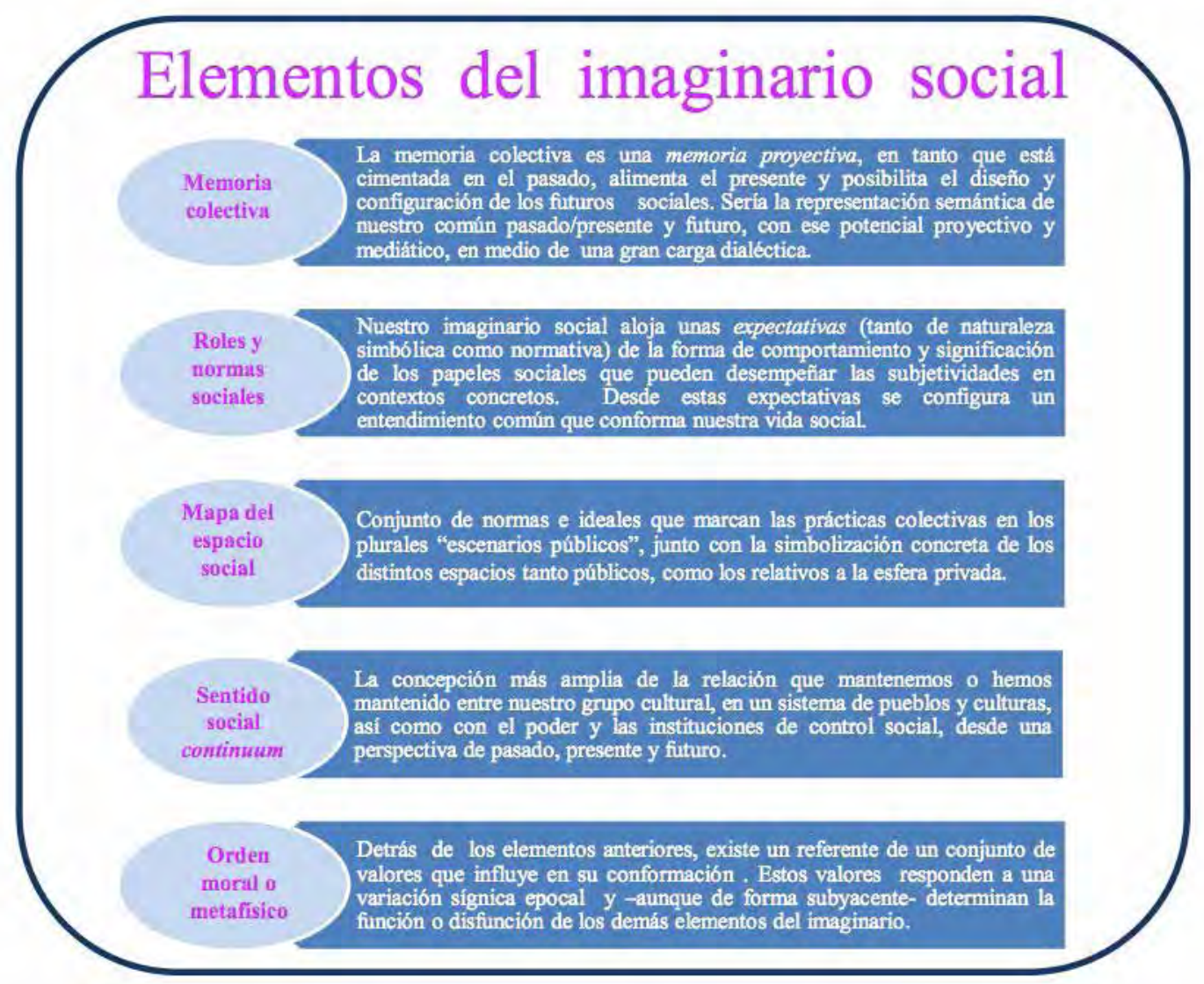

Cuadro 1: Propuesta de categorización de los principales elementos del imaginario social.

(Elaboración de la autora).

\section{Ejes conceptuales del Self Territory 174}

Esta nueva técnica etnográfica se fundamenta en cuatro conceptos principales: paisaje, su proyectabilidad sígnica y experiencia de salud, teniendo presente que la construcción de nuestras representaciones simbólicas se hace desde nuestros imaginarios sociales. Tras la delimitación conceptual del Paisaje en el apartado anterior, a continuación presentamos nuestra concepción de salud desde la experiencia de salud (Gaona, 2014) ${ }^{9}$. Entendemos la salud como ese proceso íntimo y social de producción y apropiación de sentidos, que abarca desde la intersubjetividad interpretativa sobre la enfermedad, el dolor, la vida y el cuerpo, hasta los escenarios de la medicalización la calidad de vida, la salud como capital, la salud

9 En http: //www.methaodos.org/revista-methaodos/index.php/methaodos/article/view/54 (Consultado el 20 de septiembre del 2016). 


\section{Ordenación estética del paisaje Ficha etnográfica F01_DST-174}

\section{NIVEL 1 Individual}

\section{iv delimikaciónemis [L. A. Munärià)}

1. Espacios "más represertrativos' de tu puebio/com unidad/ciudad/Willa/localidad

2. Te gusta/ No te zusas/Lo cambiarias. (NO/S/ ¿Por qué?)

3. ¿Qué le taita?

\begin{tabular}{|c|c|c|}
\hline \multirow{2}{*}{\multicolumn{2}{|c|}{ Imägenes $2 \%$ delimitacion emic Sersitivo }} & \multirow{3}{*}{$\begin{array}{l}\text { ¿Ailguna delimitacion } \\
\text { temporal sen̂alada? }\end{array}$} \\
\hline & & \\
\hline Sonidos & & \\
\hline Texturas & & \\
\hline Olores & & \\
\hline Sabores & & \\
\hline
\end{tabular}
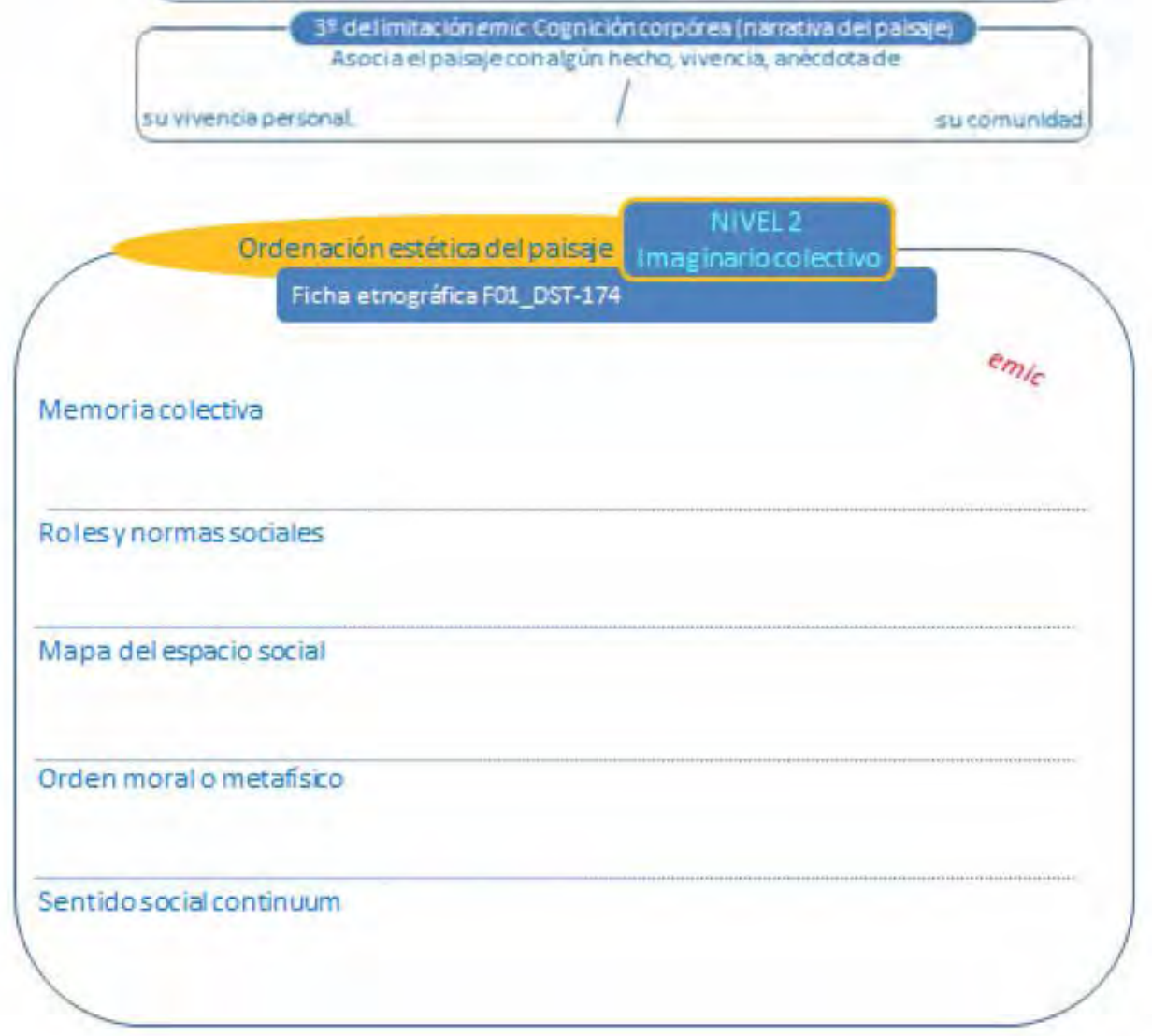

Ficha etnográfica (F01_DST174). Ordenación estética del paisaje. (Elaboración de la autora). 
como derecho, y la salud como deber, para finalizar en la percepción de la muerte. En un segundo orden, la experiencia de salud es una producción intersubjetiva de sentidos desde un contexto sociocultural concreto, un imaginario social, un lugar de continuums sígnicos, adscrita a un discurso terapéutico concreto y una retroalimentación constante con el Paisaje.

Referente al imaginario social más que una delimitación conceptual, cabe establecer una categorización de sus principales elementos que además de atender a un diseño ordenado en la recogida de datos, responde a una propuesta pedagógica de aproximación reflexiva al imaginario. En cuadro 1 se presenta dicha categorización.

\begin{tabular}{|c|c|c|}
\hline \multicolumn{2}{|c|}{$\begin{array}{l}\text { Ordenación biopolítica del paisaje } \\
\text { Ficha etnográfica F02_DST-174 }\end{array}$} & $\begin{array}{c}\text { NIVEL } 3 \\
\text { Sociodiscursivo } \\
\end{array}$ \\
\hline & \multicolumn{2}{|c|}{ LOCALIZACIÓN DE AGENTES } \\
\hline & Estructura & Historia \\
\hline \multicolumn{3}{|l|}{$\begin{array}{l}\text { LOCALES / } \\
\text { SUPRANACIONALES } \\
\text { Públicas } \\
\text { Empresariales } \\
\text { Agropecuarias } \\
\text { Sociales } \\
\end{array}$} \\
\hline \multicolumn{3}{|l|}{$\begin{array}{l}\text { NUEVOS AGRNTES } \\
\text { Públicos } \\
\text { Empresariales } \\
\text { Agropecuarios } \\
\text { Sociales } \\
\end{array}$} \\
\hline \multicolumn{3}{|r|}{ POLÍTICAS } \\
\hline & Continuidad & Innovación \\
\hline $\begin{array}{l}\text { SOBRE EL PATRIMONIO } \\
\text { Cultural histórico } \\
\text { Cultural medioambiental }\end{array}$ & $E / N-A / S$ & $E / N-A / S$ \\
\hline $\begin{array}{l}\text { SALUD } \\
\text { Del territorio } \\
\text { De la población }\end{array}$ & $E / N-A / S$ & $E / N-A / S$ \\
\hline \multirow[t]{2}{*}{ TECNO-ECONÓMICA } & $E / N-A / S$ & $E / N-A / S$ \\
\hline & \multicolumn{2}{|c|}{ PROYECTABILIDAD PAISAJE } \\
\hline & ... a corto plazo? & ...a largo plazo? \\
\hline $\begin{array}{l}\text { ¿Cómo visualizaría su pueblo } \\
\text { /comunidad/ ciudad / villa... }\end{array}$ & & \\
\hline
\end{tabular}

Ficha etnográfica (F02_DST174). Ordenación biopolítica del paisaje. (Elaboración de la autora). Leyenda: $E$ : Planes europeos. $N-A$ : Planes generales (nacionales y autonómicos). $S$ : Planes sectoriales/territoriales 


\section{Ejes metodológios del Self Territory 174}

En un primer bloque se han diseñado dos primeras fichas que buscan localizar los elementos de referencia de una comunidad sobre su paisaje, son fichas con respuestas abiertas para poder coger en las narraciones de los entrevistados los distintos y plurales sentidos que una población otorga a su paisaje. Las fichas F01_DST174 y F02_DST174 se ordenan atendiendo a una categorización de los principales elementos que conforman un imaginario social. En el análisis semántico de las representaciones recogidas se recogerá los niveles de potencialidad sígnica del paisaje; la mayor o menor presencia de ésta vendrá conformada por la constatación de representaciones colectivas creativas alejadas de los límites logocéntricos, para pasar luego después a analizar los órdenes de intencionalidad en una escala semántica jerárquica que incluye aspectos sobre prácticas saludables, niveles de cohexión social, niveles de activismo y asociacionismo, niveles de resistencia y niveles de identificación con el paisaje. Las fichas $\left(F 03 \_D S T 174\right)$ y $\left(F 04 \_D S T 174^{10}\right)$ se centran en la relación entre paisaje y experiencia de salud. En su elaboración se han tenido como referentes conceptuales los siguientes conceptos: pluralidad de espacios sociales-saludables; modelo de comportamiento ante la enfermedad (Mechanic); red profana de referencia (Good), apropiación médica adaptativa (Memel-Fôte).

Las dos últimas fichas (F03_DST-174) y (F04_DST-174) pese a tener una misma composición, tienen distinta configuración conceptual. Ambas están centradas en la ordenación semántica de la experiencia de la salud de los paisajes cotidianos de nuestra comunidad interpretativa de análisis. En ambos casos se hace una selección y análisis de cinco espacios escogidos de forma libre por el entrevistado, si bien en la ficha F03_DST-174, no se pregunta de forma directa sobre espacios de salud, pero si hay una traslación etic por parte del investigador. En este ficha, se responde a la petición de que se señalen los cinco espacios principales que conforman la cotidianeidad de su vida. Por el contrario en la ficha F04_DST-174, si se pregunta de forma directa y clara que se señalen aquellos paisajes que asocian con la salud de su comunidad o de su propia experiencia de salud.

\section{Últimas consideraciones}

A lo largo de este artículo buscamos interpelar la labor etnográfica recogiendo la tradición observacional inaugurada por la progenie boasiana o la del propio Malinowski, así como esta nueva técnica etnográfica es una continuidad en el convencimiento de no cesar en la construcción de nuevas técnicas de recogida y sistematización de los datos etnográficos. Ya que nos movemos en un abismo ontológico en el campo de la realidad sociocultural, y que la cultura en el sentido deleuziano y estoico de la palabra es un simulacro, una táctica a seguir sería la de ser científicos "elegantes" desde la definición de Alberto Cardín. En nuestra labor científica, tal y como nos enseñaba en sus clases el profesor Alberto Cardín tenemos que "seleccionar (subjetiva, o "artísticamente", si se quiere) entre una abrumadora heterogeneidad de elementos, frente a cualquier actividad, rito o acción de la sociedad observada (habla del nivel puramente gestual-accional: no digamos lo que se plantea en los niveles metalingüísticos, es decir, las explicaciones, más o menos ya elaboradas de los informantes) para construir una descripción significativa (o, si se quiere, en términos matemáticos: elegante)" (Cardín, 1990: 24).

Ser "elegantes" ante el Paisaje implica un primer vestirse desde el signo en su momento íntimo de construcción corpórea intrasubjetiva, de ahí que nuestra ficha etnográfica $\mathrm{FO1}$ DST174, recoja la narración del Paisaje desde la cognición corpórea, para pasar luego después a la construcción intersubjetiva colectiva. Ambas tienen como plantilla cognoscitiva

10 Se registran 5 fichas (F03_DST174) y 5 fichas (F04_DST174), relativas a paisajes seleccionados de forma libre por los entrevistados. 


\section{DIAGNOSIS SELF TERRITORY -174}

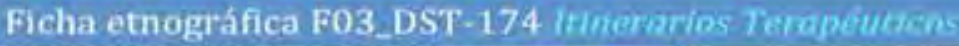

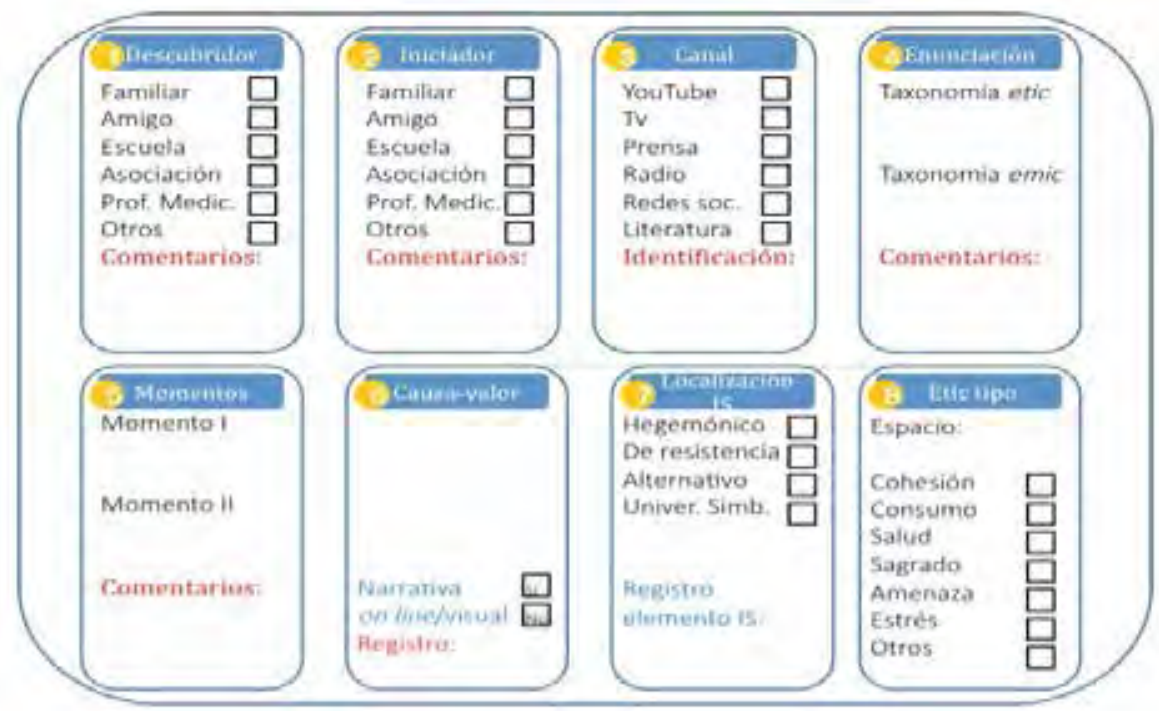

Ficha etnográfica (F03_DST-174). (Elaboración de la autora).

\section{DIAGNOSIS SELF TERRITORY -174}

Ficha etnografica F04_DST-174 Esoacios Salidables

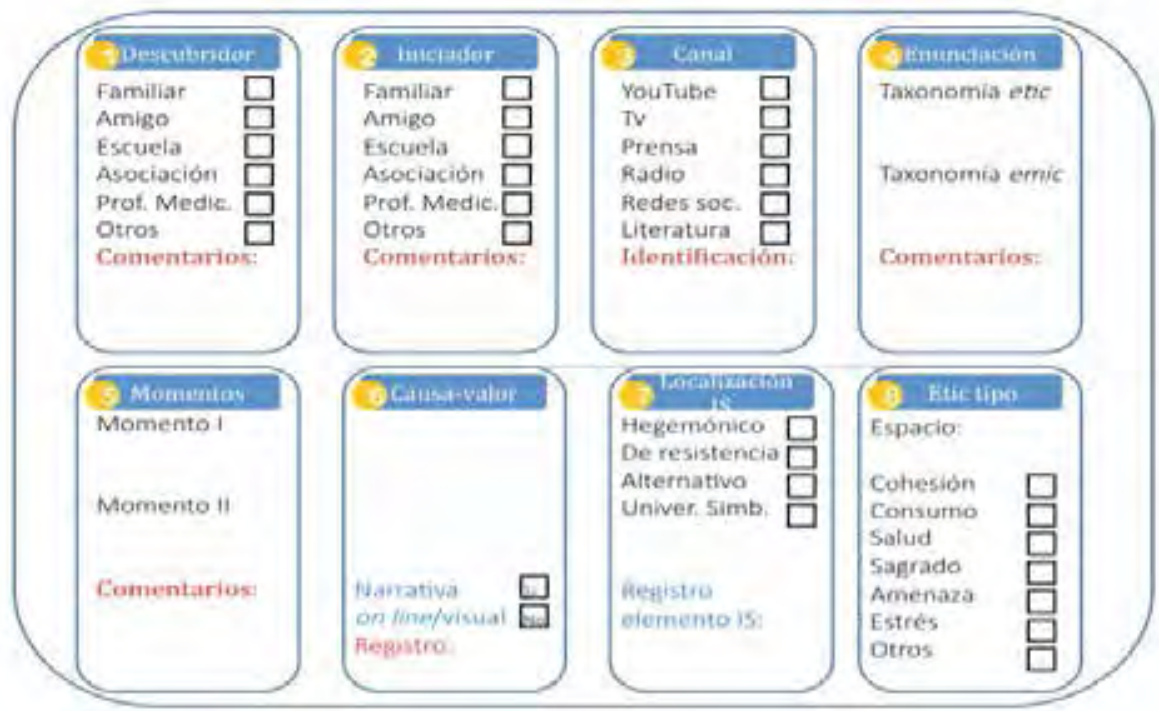

Ficha etnográfica (F04_DST-174). (Elaboración de la autora). 
de referencia el imaginario social o colectivo y constituyen una ordenación estética del paisaje. Pero en ese simulacro cultural en el que están inmersas las subjetividades, la elegancia implica también enfrentarse a vislumbrar la latente construcción discursiva del Paisaje, que se pretende quede reflejada en la segunda ficha diseñada sobre su ordenación biopolítica, en la F02_DST174. Por último en una apuesta por alcanzar esa elegancia con distinción, se elaboraron las dos últimas fichas etnográficas, centradas en la experiencia de salud de nuestras comunidades interpretativas, para que de la mano de los itinerarios terapéuticos y de los espacios saludables, vislumbremos la existencia de esa proyectabilidad del Paisaje, que teóricamente he presentado en este texto.

Como etnógrafos elegantes, estas fichas no suponen una propuesta cerrada ni excluida a reformulaciones futuras, todo lo contrario. Por otro lado, pese a un primer nivel de implementación del Método Self Territoy 174, se espera pueda contribuir -desde la sistematización de las metáforas y narraciones emic-, a vislumbrar ese azul del Paisaje. Será entonces cuando desde los lindes de la Etnografía entenderemos cómo nuestras subjetividades en interacción y diálogo estético con el Paisaje, sea del tipo que sea, proyectan la sensación de epifanía de los azules que utilizaba Joachim Patinir: "Sin duda tiene que haber quedado algo de aquel tiempo en el que todo el mundo era azul..... del azul primigenio.... del origen de la comprensión del mundo".

\section{Bibliografía}

ALVAREZ, Ester

2007 "Interpretación en arqueología: teorías del conocimiento", en Cuadernos de Arqueología de la Universidad de Navarra 15: 9-30.

2010 "Historia de la percepción y memoria cultural influencia en los estudios arqueológicos, en Cuadernos de Arqueología, (18): 173-197.

ALVAREZ MUNARRIZ, Luis

2007 "Conciencia y conducta medioambiental: los paisajes culturales" en Intersticios. Revista sociológica de pensamiento crítico, 1.

2010 "El agua como geosímbolo" en Lisón Tolosana, C. (Edt.) Agua y cultura, Zaragoza: Fundear.

2015 Categorías Clave de la Antropología. Sevilla: Signatura Demos.

ANRUBIA, Enrique y GAONA, Carmen

2008 "Epistemología del paisaje. Resignificación antropológica de la espacialidad en la montaña y en la ciudad", en Gazeta de Antropología, 2008, 24 (2).

BARRETT, John C.

1999a "Chronologies of landscapes". En Ucko, Peter J. y Layton, Robert (eds.) (1999) en The archaecology and anthropology of landscape. Shaping your landscape, Londres, Routledge, 21-30.

1999b "The mythical landscapes of the British Iron Age". En Ashmore, Wendy y Knapp, A. Bernard (eds.) Archaelogies of Landscape: Contemporany Perspectives, Oxford, Blackwell Publishers: 253-265.

BRAUDEL, F.

1968 «Aportación de la historia de las civilizaciones», en La Historia y las ciencias sociales, Madrid: Alianza Editorial, 1968: 156-184.

1963 Las civilizaciones actuales. Estudio de historia económica y social, Madrid: Tecnos.

CARAMUEL de LOBKOWITZ, J.

1984 Architectura civil recta y oblicua. Edición facsimilar, depositaba en la Biblioteca Nacional, Madrid).

CARDÍN, Alberto

1990 Lo próximo y lo ajeno. Barcelona: Ediciones Icaria.

CLAUDEL, Paul

2015 El ojo oye. Madrid: Vaso Roto 
COVARRUBIAS, F. CRUZ, G.

2011 "El problema del "sentido" de la naturaleza. En Quivera, vol. 13, núm. 2: 35-51. México.

CRIADO, BOADO Felipe

1988 "Arqueología del paisaje y espacio megalítico en Galicia”, en Arqueología Espacial, 12: 61-117.

1989 "Megalitos, espacios, pensamiento", en Trabajos de Prehistoria vol. 46P75-98.

CRUZ, Sor Juana Inés de la

2000 Poesía Lírica. José Carlos González Boixo (ed.). Madrid: Cátedra.

DANIELS, Stephen y COSGROVE, Denis

1988 "Introduction; iconography and landscape". En Cosgrove, Denis y Daniels, Stephen (eds.): The iconography of landscape. Essays on the symbolic representation, design and use of past environments, Nueva Yord, Cambridge, University Press, pp.1-10.

ELLARD, Colin.

2016 Psicogeografía. La influencia de los lugares en la mente y en el corazón.

GAONA, C.

2009 Ágora del impresionismo sociocultural, Ed. Dykinson, Madrid.

2014 "Sujetos del dolor, territorio de la dignidad", en Revista Methaodos, Vol.2 Núm. 2: 227249.

GLENBERT, A, VEGA, M. y GLAESSER, A:

$2008 \quad$ Symbols and Embodiment, Oxford: U.P.

GOSDEN, Chris y LOCK, Gary

1998 "Prehistoric histories", en World Archaecology, 30 (1) (The past in the pasa: the reuse of ancien monuments), 2-12.

HOLTORF, Cornelius

2000 Monumental past: the life-histories of megalithic monuments in Mecklenburg-Vorpommern (Germany) (Monografía electronica en constante actualización) Toronto: Centre for Instructional Technology Development. En: https: //tspace.library.utoronto.ca/citd/holtorf/ index.html

JOHNSON, Mark

1991 El cuerpo en la mente: Fundamentos corporales del significado la imaginación y la razón. Madrid: Debate.

KAHN, Miriam

1996 "Your place and mine: sharing emocional landscapes in Wamira, Papua New Guinea". En Feld, Steven y Basso, Keith H. (eds.): Senses of place, Santa Fe School of American Research: 167-196.

KNAPP, Arthur Bernad y ASHMORE, Wendy

1999 "Archaeological landscapes: constructed, conceptualized, ideational", en Ashmore, Wendy y Knapp, A. Bernard (eds.): Archaelogies of Landscape: Contemporary Perspectives, Oxford: Blackwell Publishers: 1-33.

LACHAUD, Jean-Marc y NEVEUX, Olivier (ed.).

2007 "De la dimension crítica de cuerpos en acción en el arte contemporáneo" en Lachaud, J.M. y Neveux, Ol. Cuerpos dominados. Cuerpos en ruptura, Ed. Ediciones Nueva Visión, Buenos Aires, pp.95-114.

LAFFON DE LA ESCOSURA, Carmen

2000 Visión de un paisaje. Discurso de ingreso en la Real Academia de Bellas Artes de San Fernando. Madrid

LASCH, Christopher

1991 The Culture of Narcissism: American Life in an Age of Diminishing Expectations. Nueva York: W.W. Norton\&Company; trad. cast: 1999, La cultura del narcisismo. Barcelona: Andrés Bello.

LE BRETON, D.

1995 Anthropologie du corps et modernité. París: PUF

LEFEBVRE, Henri

1974 La production de l'espace. París: Anthropos. 
MADERUELO, Javier

1997 "Introducción: el paisaje”. En Maderuelo, Javier (dir.): El paisaje: arte y naturaleza, Huesca, Diputación de Huesca: 9-12.

MARTÍNEZ DE PISÓN, Eduardo

2002 "Reflexiones sobre el paisaje". En Ortega, Nicolás (ed.): Estudios sobre historia del paisaje español, Madrid, Universidad Autónoma de Madrid: 13-24.

MERLEUA-PONTY, M.

$1975 \quad$ Fenomenología de la percepción. Barcelona: Península

NORA, Pierre

1984 Les lieux de mémoire, vol. 1, París, Gallimard

PAPALINI, Vanina

2015 Garantías de felicidad. Estudio sobre los libros de autoayuda. Buenos Aires: Adriana Hidalgo.

PARCERO, César, CRIADO, Felipe y SANTOS, Manuel

1998a "La arqueología de los espacios sagrados", en Arqueología Espacial (19-20): 507-516.

PARCERO, César

1998b "Rewriting landscapa; incorporating sacred landscapes into cultural traditions", en World Archaecology 30 (1): 159-178.

ROSE, Nikolas

$2012 \quad$ Políticas de la vida. Buenos Aires: Unipe.

SALDARIRIAGA, Patricia

2006 Los espacios del Primero sueño de Sor Juan Inés de la Cruz. Arquitectura y cuerpo femenino. Madrid: Iberoamericana-Vervuert

SENNET, Richard

2000 La corrosión del carácter. Barcelona, Ed. Anagrama

1997 Carne y piedra: el cuerpo y la ciudad en la civilización occidental. Madrid: Alianza Editorial.

TORREGROZA, Enver Joen

2008 "Del viajero al turista: estética y política del paisaje urbano", en Rev. Desafíos, (19): 71103.

ZOIDO, Florencio

2012 El paisaje un concepto útil para relacionar estética, ética y política. Scripta Nova. Revista Electrónica de Geografía y Ciencias Sociales. Barcelona: Universidad de Barcelona, 10 de julio de 2012, vol. XVI, no 407. En http: //www.ub.es/geocrit/sn/sn-407.htm

2010 "Territorio y paisaje, conocimiento, estrategias y políticas" en PILLET, F. y CAÑIZARES, C. Territorio, paisaje y sostenibilidad. Barcleona: El Serbal: 87-114.

2011 "El paisaje un concepto útil para relacionar estética, ética y política", en Scripta Nova. Universidad de Barcelona, vol. XVI, núm. 47.

2006 Landscape and spatial planning policies. En Landscape and sustainable development: challenges of the European Landscape. Convention Estrasburgo. Consejo de Europa

VIRNO, Paolo

2013 Y así sucesivamente al infinito. Lógica y antropología. Buenos Aires: Fondo de Cultura Económica de Argentina.

WATSUJI, Tetsuro

2006 Antropología del paisaje. Climas, culturas y religiones. Salamanca: Sígueme.

WILSON, M.

2002 "Six Views of Embodied Cognition" en http: //philosophy.wisc.edu/shapiro/ PHIL951/951articles/wilson.htm consultado el 20 de julio del 2014. 